\title{
Les campus numériques en France : réalisations, dynamiques et émergences
}

Virtual campuses in France: realisations, dynamics and emergences

Julien Deceuninck

\section{(2) OpenEdition}

Journals

Édition électronique

URL : http://journals.openedition.org/edc/617

DOI : $10.4000 /$ edc. 617

ISSN : 2101-0366

Éditeur

Université Lille-3

Édition imprimée

Date de publication : 1 décembre 2007

Pagination : 173-192

ISBN : 978-2-9514961-8-7

ISSN : $1270-6841$

Référence électronique

Julien Deceuninck, "Les campus numériques en France : réalisations, dynamiques et émergences », Études de communication [En ligne], Numéro spécial | 2007, mis en ligne le 01 octobre 2009, consulté le 30 avril 2019. URL : http://journals.openedition.org/edc/617 ; DOI : 10.4000/edc.617

Ce document a été généré automatiquement le 30 avril 2019

(c) Tous droits réservés 


\title{
Les campus numériques en France : réalisations, dynamiques et émergences
}

Virtual campuses in France: realisations, dynamics and emergences

\author{
Julien Deceuninck
}

\section{Introduction}

1 Un des objectifs de l'ERTe, cela a déjà été rappelé, était de voir en quoi les expériences d'introduction du numérique dans l'enseignement supérieur avaient en perspective un débouché sur des "modèles économiques, organisationnels, institutionnels tenables dans la durée $»^{1}$. A la lumière de l'étude menée, il se confirme que le monde de l'enseignement public n'est guère le lieu d'une ingénierie classique où les choix se feraient à partir d'analyses de coûts et en fonction de critères explicites et débattus dans les structures de pilotage du projet. On se trouve encore face à une ingénierie d'émergence, de nature «constructiviste» (Deceuninck, Laisne, 2004). Plus précisément, l'économie de ces dispositifs se construit en même temps que les dispositifs eux-mêmes.

2 Rendre compte des expérimentations qu'ont été les campus numériques nécessite ainsi de rendre compte de dynamiques complexes où acteurs, objets techniques, représentations «bougent » sans cesse. Les dispositifs qui se sont développés dans le cadre des appels à projets relevaient d'une situation d'incertitude maximale. Incertitude sur les attentes des autorités de tutelle : les Campus numériques sont venus après d'autres appels à projets et se sont eux-mêmes trouvés rapidement dépassés par les nouveaux projets d'Universités Numériques Thématiques et d'Universités Numériques Régionales aux objectifs nettement moins liés à la proposition d'une offre de formation en ligne au sens strict. Incertitude aussi sur les valeurs de référence : fallait-il viser une rationalisation, voire une rentabilisation de l'offre de formation? S'agissait-il de faire preuve d'inventivité et de créativité ? Ou encore fallait-il chercher une aide à la réussite pour un plus grand 
nombre? De fait, les mondes civique, industriel ou encore artiste évoqués par Luc Boltanski et Laurent Thévenot pouvaient être et furent convoqués selon des degrés variables dans la plupart des projets. Dans un tel contexte, le jeu des acteurs semble prépondérant: en effet, les conjonctions locales d'acteurs, les représentations de ces acteurs, leurs positions idéologiques sont nécessairement à convoquer pour expliquer les caractéristiques des différents dispositifs de formation de l'opération.

3 Au-delà de ce caractère mouvant, nous renvoyons ici aux acteurs une série de réflexions et d'interrogations sur les dynamiques qui peuvent s'installer dans et au-delà de l'action. Pour ce faire, nous centrant uniquement sur des dispositifs de formation diplômants, nous proposerons deux approches complémentaires des campus numériques. La première tend à montrer qu'ils se sont inscrits dans une dynamique plus large qui traverse le champ universitaire dans son ensemble, indépendamment de l'appel d'offre ministériel et qui relève des modes de pensée traditionnels de la culture universitaire. La seconde montre que, à travers les campus, se profile en même temps la question des nouveaux types d'équilibre d'ordre info-communicationnel dans les dispositifs de formation.

\section{Des réalisations contrastées dans une dynamique générale du champ universitaire}

\section{La diversité de destins des campus numériques}

4 Le premier point sur lequel nous voulons attirer l'attention est que les campus ne sont pas des éléments isolés mais qu'ils s'intègrent dans une dynamique du champ universitaire qui dépasse le cadre des appels à projets lancés en 2000 et 2001. Pour mieux comprendre la dynamique propre des campus, il faut donc observer aussi ce qui a pu émerger simultanément dans le champ en dehors de ces projets spécifiques.

Quand on regarde le paysage des formations universitaires françaises présentes sur l'Internet du point de vue des solutions techniques, on constate que celles-ci se sont développées dans de nombreuses universités selon un rythme proche. La question des plates-formes a suscité beaucoup de débats au début des années 2000, mais c'est essentiellement le CNED qui a fait l'expérience d'outils lourds et coûteux. Les universités, pour leur part, se sont progressivement dotées d'outils plus ou moins similaires relevant du domaine du «libre». Quant aux fonctions des plates-formes, elles sont sensiblement les mêmes d'un établissement à l'autre : dépôt et téléchargement de fichiers, éléments de communication inter-individuelle ou collective de type courrier électronique, forum ou chat. Les agencements peuvent varier selon les campus mais aucun d'entre eux n'a donné naissance à une plate-forme résolument différente des autres, radicalement novatrice sur le plan de la communication ou des modalités d'enseignement. Les solutions techniques sont donc toutes très proches les unes des autres et la question majeure n'est pas d'ordre technique.

6 Concernant la nature des dispositifs et leur usage réel dans l'univers des formations proposées par les universités, l'offre qui s'est construite progressivement est diverse et contrastée. Nous ne nous sommes pas livré à une analyse exhaustive (il y avait plus de 70 projets) mais les campus analysés dans le cadre de l'ERTe illustrent bien cette situation. F. Horn et T. Lamarche, par exemple, constatent que le campus UVMF se révèle être un portail dont les usages n'ont pas réellement fait l'objet d'une évaluation en termes 
d'impact sur les dispositifs de formation du monde médical. P. Mœglin à propos de l'UeL et de C@mpusciences montre quant à lui que les ressources réalisées par les enseignants peuvent « avoir plusieurs vies » mais que, dans aucune de celles-ci, l'usage en formation n'est réellement avéré ou du moins n'atteint une ampleur significative d'une mutation réelle du mode d'enseignement. Ces deux projets relèvent d'un souci de développer des ressources pédagogiques accessibles en ligne, mais non de la volonté de mettre en place des dispositifs de formation au sens strict, débouchant sur une certification ou une validation institutionnelle.

7 Pour les campus qui se sont placés d'emblée dans une perspective de formation diplômante, on ne peut que constater également la même diversité des résultats. Après bien des vicissitudes, le projet CampusCultura, (Fichez, Benchenna, 2005) a momentanément trouvé un équilibre. En économie et gestion, Canège, longuement analysé par P. Grevet (2005), a éprouvé des difficultés à trouver un public suffisant pour assurer les retours espérés en termes financiers. Dans le secteur des sciences de l'éducation, le campus Forse (Deceuninck, 2005), de son côté, a réussi à se stabiliser. Certains campus n'ont tout simplement pas vu le jour. D'autres se sont métamorphosés. Quelques uns, peu nombreux, ont réussi à de maintenir sous la forme annoncée par leurs promoteurs.

\section{Le cas des préparations au Diplôme d'Accès aux Études Universitaires (DAEU)}

8 La diversité des domaines de formation couverts par les campus numériques ne facilite pas les comparaisons. Quelques domaines le permettent cependant comme celui de la formation débouchant sur le DAEU qui a fait l'objet de deux projets de campus : Pégasus et Passeport pour l'Université . Des deux projets initiaux, seul le premier est aujourd'hui totalement opérationnel ${ }^{3}$. Le second n'a pas abouti à une offre unique de formation en ligne : deux des établissements partenaires affichent en effet aujourd'hui chacun de leur côté leur propre offre de formation et, parmi les autres membres du consortium initial, cinq universités partenaires d'une même région qui leur avait octroyé des financements régionaux complémentaires, offrent bien aux enseignants la possibilité d'accéder à des ressources communes à partir d'une plate-forme, mais ne proposent pas une formation en ligne à laquelle les étudiants pourraient s'inscrire directement. Et enfin, parallèlement aux dispositifs liés à l'appel à projet Campus numériques, d'autres universités proposent aujourd'hui une offre propre sur ce segment de formation ${ }^{4}$.

9 La préparation au DAEU relève de la formation continue et concerne un public important qui est réparti sur l'ensemble du territoire. Elle vise un niveau d'étude clairement défini, même si chaque établissement est libre de définir le contenu précis des modules et les modalités d'évaluation. En outre, dans certains cas, des coordinations régionales interuniversitaires ont harmonisé un cadre général pour les enseignements. Dans un tel contexte, on aurait pu penser que les dispositifs allaient relever d'une mutualisation des ressources de formation et viser, au niveau régional ou local, les publics éloignés des centres universitaires. Mais les dynamiques ont été différentes: la mutualisation de ressources élaborées de manière collaborative ne s'est pas faite.

10 A titre d'explication du faible développement des campus numériques dans ce domaine, on a pu mettre en avant des problèmes tarifaires. Dans le secteur, en effet, les tarifs sont définis en termes d'heures/stagiaire et les frais pédagogiques des demandeurs souvent pris en charge par des organismes de financement ou par les collectivités territoriales, 
tandis que les organismes de formation sont contraints de reverser les heures non assurées par un enseignant ou non suivies par l'étudiant. Certains acteurs avancent que la Loi n'autoriserait de financement public que pour la formation présentielle et que la formation sur l'Internet ne pourrait ainsi pas être prise en compte. D'autres acteurs cependant considèrent qu'il n'y a pas de problème, que les formations à distance peuvent être financées au même titre que les autres, et certains dispositifs proposent de fait à la fois une formation traditionnelle en cours du soir et une formation à distance. La réglementation en vigueur n'est sans doute pas facilitatrice, mais elle n'explique pas tout et, selon les cas, les acteurs parviennent à s'en accommoder.

11 L'appel à projet Campus numériques a donc bien suscité la mise sur pied d'un dispositif, Pégasus, qui se maintient dans la durée, mais on peut estimer que l'ensemble des dispositifs mis en place dans le domaine du DAEU relève d'une dynamique endogène propre à la formation continue universitaire. L'appel à projet a été une opportunité pour poursuivre des projets latents ou déjà partiellement mis en œuvre et le consortium, qui était un des critères majeurs des Campus numériques, n'a pas réussi à se constituer comme espace de légitimité pour le DAEU: la tendance à la concurrence entre établissements ${ }^{5}$ l'a emporté sur les velléités de mutualisation.

\section{Le cas des formations en sciences de l'éducation}

12 Dans le domaine des sciences de l'éducation, nous nous sommes attaché de manière plus approfondie à un campus qui apparaît comme une réussite au regard des objectifs initiaux des appels à projet : le campus Forse (Formation en Sciences de l'Éducation) porté par les Universités de Rouen et de Lyon 2 et par le CNED. Celui-ci apparaît aujourd'hui comme une structure qui, selon ses animateurs, a trouvé un équilibre financier et peut désormais se développer indépendamment des subventions initiales. Il propose des formations de niveau $\mathrm{L}$ et $\mathrm{M}$ en sciences de l'éducation : licence, maitrise, DESS (qui ont évolué en master professionnalisant), et master de recherche à distance, visant un public francophone ouvert à la rentrée 2004-2005. Le campus touche un public de plus d'un millier d'étudiants, relevant à la fois du cadre de la formation initiale et de celui de la formation continue, les effectifs du premier l'emportant cependant très largement.

13 La réussite du projet et sa viabilité économique et organisationnelle semblent tenir en grande partie au fait que ce campus numérique a pris appui sur un dispositif de formation à distance qui existait préalablement sous la forme d'un partenariat entre l'université de Rouen et le CNED. Dans ce contexte, l'engagement du projet s'est fait essentiellement en termes de dispositif et de distance. Les contenus d'enseignement existaient déjà sous forme papier pour les niveaux Licence et DESS et de ce fait, les financements obtenus ont essentiellement été consacrés à la médiatisation d'un certain nombre de modules complémentaires et à l'ouverture d'une maîtrise, ce qui a permis de proposer un cycle complet de formation.

Le campus Forse a trouvé son équilibre dans une double complémentarité. La première se situe dans le cadre de la formation initiale et s'établit entre, d'une part, une formation de masse, relativement traditionnelle et à faible coût, au niveau de la licence (entre 800 et 900 étudiants chaque année) et, d'autre part, une formation individualisée, innovante mais beaucoup plus coûteuse, portant sur des effectifs volontairement limités en nombre, au niveau de la maîtrise (ou master 1). Cette dernière formation, qui propose notamment aux étudiants du travail collaboratif à distance, présente pour sa part un caractère plus 
expérimental sur le plan pédagogique. Une seconde complémentarité est réalisée entre formation initiale et formation continue au niveau du master 2 professionnel. Les tarifs plus élevés de la formation continue et le nombre d'étudiants plus important qu'en master 1 se traduisent par un apport financier non négligeable. En croisant ainsi les différentes sources et les différents types de financement, le dispositif trouve un équilibre global. L'expérience accumulée dans le dispositif de formation à distance préexistant à la réponse à l'appel à projets n'a certes pas évité aux acteurs de se trouver confrontés à des ajustements nécessaires en ce qui concerne les modalités financières du partenariat, tant sur le plan de la prise en compte des recettes et des dépenses que sur celui de la redistribution entre les partenaires des excédents dégagés ${ }^{6}$. Mais, cette épreuve a finalement pu être surmontée par un avenant à la convention partenariale réglant la question du mode de prise en compte des frais de structure.

Enfin, la réussite du projet tient sans doute aussi à une spécificité du domaine des sciences de l'éducation. Celui-ci est marqué en effet par une forte demande de formations diplômantes, demande à laquelle l'offre des universités des différentes régions n'apporte pas de réponse ou n'apporte qu'une réponse partielle. La formation à distance n'a pas à chercher son public. Le campus Forse est donc susceptible de se maintenir dans la durée, avec des ajustements certes, mais il le fera tant que la demande de formation en sciences de l'éducation restera élevée et que d'autres opérateurs ne se présenteront pas pour le concurrencer.

On dispose donc ici d'un cas dont on pourrait tirer des enseignements dans la perspective du développement d'autres campus. Ici encore, cependant, il faut noter que d'autres dispositifs ont atteint des résultats identiques en dehors de l'appel à projet du ministère. Des Centres de Télé-Enseignement Universitaires (CTEU) offrent également des dispositifs de formation à distance aux niveaux Licence et Master. Plusieurs autres ont même développé leurs propres plates-formes en dehors de tout soutien extérieur. On constate donc que, comme dans le cas du DAEU, l'enseignement supérieur est traversé par une dynamique de l'enseignement numérique endogène. L'idée d'une péréquation globale entre éléments d'une formation n'est d'ailleurs pas non plus une spécificité du campus : la plupart des CTEU fonctionnent selon cette logique, certaines formations aux effectifs très nombreux permettant de maintenir l'offre de formations largement déficitaires.

\section{Une intégration générale dans la culture universitaire traditionnelle}

17 Dans les dispositifs comme Forse, les acteurs ont surtout été guidés par la volonté d'assurer la mise en œuvre d'une formation diplômante effective et non de développer des éléments partiels, de type expérimental (quelle que soit la nature de l'expérience, technique ou pédagogique). Les dispositifs qui se maintiennent sont en fait caractérisés par le côté pragmatique du projet originel et le souci d'être sûr que la formation sera assurée dans son intégralité et débouchera sur le passage du diplôme ${ }^{7}$.

L'inscription institutionnelle des campus présente à la fois des similitudes et des différences par rapport aux autres dispositifs de formation à distance. Dans le cas des Centre de télé-enseignement et Services Universitaires de Formation Continue, le statut est clairement défini: il s'agit de services communs. Ces services cependant, contrairement aux services informatiques ou aux services audiovisuels, ont la particularité de générer des ressources financières, ce qui leur permet d'avoir une certaine autonomie dans l'établissement. Le système de débat et vote par les conseils est 
certes respecté, mais le plus souvent sans qu'il soit contraignant sur le développement des services et des projets.

Dans le cas des campus, la situation est différente. Certes ce sont les Présidents qui ont la responsabilité officielle des projets, mais, le plus souvent, ils délèguent très largement la conduite de ceux-ci à des chefs de projet dont les liens avec les autres structures (UFR et services communs) ne sont pas définis avec précision. Nous avons qualifié de "circonscrit» ce mode de management: une zone de liberté est créée au sein de l'établissement et c'est cela qui permet le développement des initiatives. La contre-partie est que l'équipe projet n'est pas assurée de la même pérennité que les services communs. Le caractère inter-universitaire et les partenariats avec le CNED dans le cadre des consortiums renforcent encore cette spécificité qui les fragilise.

En ce qui concerne la répartition des rôles dans les campus, les configurations restent très proches des schémas universitaires traditionnels. La fonction de chef de projet est à peine reconnue sur le plan institutionnel. Il n'y a pas de fonctions nouvelles pour la médiatisation des contenus. L'externalisation est restée faible et la production des ressources est essentiellement réalisée au sein des universités (Centres audiovisuels, Centres de Ressources Informatiques...) ou du CNED. Les enseignants continuent à déterminer le contenu scientifique et pédagogique des ressources et à définir le contrôle des connaissances. Les examens gardent la forme traditionnelle de devoirs écrits en présentiel. Les éléments nouveaux sont sans doute le fait que les enseignants n'assurent plus une part importante des prestations en face à face qui sont, de fait, très réduites et le recours à des personnels contractuels ou vacataires, étudiants en thèse ou autres, pour assurer le tutorat. Si celui-ci relève d'un cadrage réglementaire précis, la question de l'intégration des nouvelles activités d'enseignement dans le service des enseignants n'a pas progressé et reste soumise à des décisions et des interprétations locales. L'apport le plus notable serait ici la notion de contrat d'auteur apportée par le partenariat avec le CNED.

21 La diversité des destins des campus numériques n'a rien d'étrange quand on la situe dans la culture du monde universitaire. Dans celle-ci, les acteurs qui répondent à un appel d'offre s'engagent plus sur un principe d'action que sur une visée de résultats; plus intéressés par l'innovation et le développement de prototypes que par la généralisation de dispositifs, ils sont plus proches du monde de l'expérimentation et de la recherche que de celui de l'ingénierie et de l'administration. Mais, en fait, l'analyse des campus demanderait à être recadrée dans un temps plus long que celui des deux ou trois années dans lesquelles se sont inscrits les appels d'offre : les dispositifs de formation qui ont vu le jour relèvent en effet du développement dans le monde universitaire d'une nouvelle culture de pilotage par projets, lesquels sont limités dans le temps, avec désengagement annoncé de l'État. Un tel changement ne relève pas d'une temporalité courte.

\section{Perspectives}

\section{Les campus entre marchandisation et industrialisation}

\section{Mise en concurrence et création d'un marché : une dynamique marchande?}

Un des objectifs de l'appel à projet Campus numérique au début des années 2000 était le développement dans les établissements d'enseignement supérieur de la capacité à 
inventer des dispositifs financièrement auto-suffisants. Ceci a pu être interprété comme une tendance à la marchandisation de la formation ${ }^{8}$. En fait, même si les tarifs que proposent des campus comme Forse sont plus élevés que ceux de l'enseignement à distance traditionnel, ceux-ci se situent pratiquement hors marché, du moins hors d'un marché qui serait réellement concurrentiel. En dehors de ce campus, trois autres universités proposent des formations à distance en sciences de l'éducation dans le cadre de la Fédération Interuniversitaire de l'Enseignement à Distance (FIED) et touchent elles aussi plusieurs centaines d'inscrits. On pourrait considérer qu'il y a bien là un marché concurrentiel, mais la demande sociale de formation diplômante dans ce secteur est telle que la concurrence ne semble pas jouer encore à ce stade. Sans doute est-ce aussi lié à d'autres considérations comme la proximité (liée aux examens obligatoirement passés dans l'université d'inscription) ou à la méconnaissance de l'existence de l'ensemble des dispositifs par le public ${ }^{9}$. Sur ce dernier point, on peut penser que les projets ultérieurs d'Universités Numériques en Région visant à rationaliser, sinon l'offre, du moins l'information sur l'offre, joueront davantage dans la constitution d'un marché sur le plan régional ; mais ils peuvent aussi être vus comme une volonté de rationalisation ou d'aide à l'étudiant dans le choix de ses études. Les Universités Numériques Thématiques, quant à elles, peuvent avoir davantage un effet de construction d'un marché national en proposant une entrée unique à l'étudiant à la recherche d'une formation, si elles ne se limitent pas à une simple offre de ressources gratuites ${ }^{10}$.

Les dispositifs de formation à distance ne relèvent pas par ailleurs d'une ingénierie qui reposerait sur une comptabilité analytique rigoureuse. On ne peut donc parler à leur propos d'une logique économique qui s'imposerait aux acteurs, même si ceux-ci négocient les évolutions du dispositif en prenant en compte le volume des financements dont ils peuvent disposer. Sur le plan des représentations, les acteurs restent dans une sorte d'artisanat instrumenté, de petite production marchande en secteur public. Concernant le campus étudié, les tarifs d'inscription demandés aux étudiants pour les frais pédagogiques de la formation à distance sont certes plus élevés que les tarifs universitaires classiques (auxquels ils viennent d'ailleurs s'ajouter), mais en l'absence de documents chiffrés, il est impossible de dire précisément quelle est la part prise en charge par les usagers du dispositif et quelle est celle qui est assurée par le financement public. Enfin, en l'absence d'une offre privée concurrente soumise aux nécessités de retours sur investissements qui donnerait des éléments de comparaison, il est impossible de dire quel est le degré réel de rentabilité du campus.

Le recours à l'économique en tant qu'argument dans les négociations et la construction des dispositifs est ici indissociable de la dynamique organisationnelle et des accords et compromis qui l'accompagnent. Lorsque les acteurs se décident à porter la controverse sur le terrain économique, ce n'est pas, du moins pour l'instant, directement pour des questions d'ordre économique, mais essentiellement pour des questions qui relèvent de l'organisationnel au sens où l'entend la sociologie de l'action organisée, c'est-à-dire de luttes d'influence ou de pouvoir au sein du réseau d'acteurs constitué autour du projet.

\section{Ressources humaines et choix technico-pédagogiques : une dynamique industrielle?}

Dans le cas de Forse, le choix d'un recours limité aux solutions multimédias en licence se traduit par des coûts fixes peu élevés. Les activités de tutorat y sont également modestes et donc peu coûteuses. La licence se révèle ainsi particulièrement excédentaire en 
matière de recettes. A contrario, les modules d'initiation à la recherche mis en place à ce même niveau dans le cadre de l'université de Lyon 2 ont posé problème : la nécessité de recourir à des formateurs qualifiés en quantité plus importante est apparue comme une source de tensions parce qu'elle augmente les coûts de la formation. De même, les responsables du projet ont fait état des difficultés à développer le dispositif au niveau master en termes de ressources humaines car, dans ce cas, il ne s'agit pas d'un tutorat peu qualifié mais d'un véritable encadrement pédagogique, voire d'une direction de recherche. Le modèle économique d'ensemble ne risque-t-il pas ainsi de contraindre les formations de niveau licence à rester dans un cadre pédagogique transmissif traditionnel proche de ce qu'était l'enseignement par correspondance?

Sur un autre plan, alors qu'elles n'ont pas encore trouvé de cadrage réellement précis, les nouvelles activités de travail collaboratif sont victimes de leur succès. Les comportements des étudiants ayant tendance à évoluer, elles deviennent à la fois plus nombreuses et plus exigeantes. Les questions posées par courrier électronique ou sur les forums s'accroissent et les tuteurs estiment que le temps de travail nécessaire pour répondre augmente également et demandent une redéfinition du mode de rémunération. La tentation est alors de limiter le tutorat. Cette tendance se retrouve dans d'autres dispositifs, avec, ici encore, la perspective de déboucher en définitive sur une forme d'enseignement proche de l'enseignement par correspondance dans lequel les plates-formes serviraient essentiellement de vecteur de documents numériques.

Enfin, au-delà des pionniers qui se sont investis dans le lancement du projet, il semble difficile de trouver des tuteurs pour les formations de niveau master et d'élargir l'offre faute d'encadrement pédagogique par des Maîtres de conférences ou des Professeurs d'université. On se trouve ici face à un véritable goulot d'étranglement, la question des ressources humaines évoluant beaucoup plus lentement que celle de la technique. Dans ce cadre, on est donc face à une dynamique industrielle classique, tendant à réduire la part du travail vivant en vue d'assurer une rentabilité maximale et la technologie numérique apparait ici comme l'opportunité technique d'un projet qui n'est pas consciemment visée par les acteurs au départ du projet. C'est pour cela que nous parlons de dynamique de système, dynamique qui se construit progressivement dans l'action et qui dépasse les intentions des acteurs, et non de logique animant les acteurs ou servant de référence au système.

\section{Perspectives d'ordre info-communicationnel}

Nous voudrions, pour terminer, poursuivre la réflexion en nous focalisant sur les dimensions informationnelles et communicationnelles, en posant plus particulièrement cette question : comment évoluent ou peuvent évoluer les rapports entre documents et échanges (entre enseignants et étudiants), ou encore, comment leur part respective estelle susceptible de se combiner en une nouvelle forme d'économie infocommunicationnelle du dispositif de formation?

Nous proposons ces deux termes dans la mesure où la terminologie employée par les acteurs reste actuellement très diverse ${ }^{11}$. Pour notre part, considérant qu'il s'agit dans tous les cas d'informations mises sur un support, de quelque nature que soit ce dernier, nous proposons de parler tout simplement de document. A côté de ceux-ci, les échanges langagiers représentent une part essentielle du « travail vivant » dans l'enseignement de face à face traditionnel. La fonction d'échange y est centrale. Dans la formation à 
distance, de même, on retrouve toujours une conjonction de documents et d'échanges. Leur articulation relève d'une forme d'économie info-communicationnelle du dispositif. Les différents projets qui ont été menés dans le cadre des Campus numériques ont tous eu en commun un horizon de référence essentiel, celui de vouloir faire passer l'enseignement par des plates-formes, c'est-à-dire par des machines et, plus précisément, par des machines en réseau puisque c'est bien ce que recouvre l'Internet. Techniquement, cependant, au niveau des représentations qu'en donnent les acteurs, il semble qu'on puisse distinguer deux pôles qui opposeraient «machines à diffuser des documents » et "échanges par réseaux de télécommunication». Nous rangeons dans une perspective « réseaux de télécommunication » les acteurs qui sont avant tout sensibles aux échanges (entre enseignant et étudiants, entre étudiants) dans le dispositif de formation. Dans la perspective " machines à diffuser", se trouvent plutôt convoqués des acteurs qui portent le regard sur le traitement de l'information en terme de médiatisation des contenus d'enseignement.

\section{Médiatiser les savoirs sous forme de documents}

Quelles sont les incidences sur l'enseignement de la médiatisation vue à travers le prisme de la «machine à diffuser »? La première, évidente, reste massivement le passage de l'oral à l'écrit. Le recours au son, à la vidéo ou aux questionnaires interactifs est présent mais reste encore secondaire. Les dispositifs proposent encore le plus souvent des documents textes sous de forme de fichiers, par exemple de type PDF, pour éviter leur modification. Parfois ils peuvent au contraire être très complexes et très coûteux quand ils apparaissent sous la forme de documents multimédias ou d'hyper documents, et doivent répondre à un certain nombre de contraintes supplémentaires liées à la compression des images et du son, aux possibilités de bande passante lorsqu'il s'agit de cours filmés, etc. Dans tous les cas cependant, on transforme du discours en document.

Quelles sont maintenant les incidences des choix de médiatisation des contenus informationnels sur la forme des dispositifs? Un certain nombre de campus ont joué la viabilité du dispositif contre l'hypermédiatisation. Selon les cas, il peut s'agir de choix volontaires dès le départ, les acteurs souhaitant avant tout mener à bien leur projet de mise à distance d'une formation globale. Dans d'autres, c'est l'insuffisance du budget qui est mise en avant pour expliquer le recours massif au « fichier texte ». On peut être aussi face à un manque de compétences techniques de la part des enseignants qui ne maîtrisent pas les outils nécessaires aux animations complexes ou qui manquent de temps pour le faire dans les délais impartis par le projet. On a également des cas où les enseignants ne peuvent ni assurer eux-mêmes les développements, ni faire appel à des développeurs privés, dans la mesure où les tarifs pratiqués par ces derniers sont beaucoup trop élevés par rapport aux financements disponibles.

\section{Communiquer avec l'étudiant}

Mettre l'accent sur la dimension communicationnelle amène à se poser d'autres questions. Il est certes réducteur de limiter la communication au relationnel et de renvoyer l'informationnel aux seuls contenus d'enseignement. C'est le point de vue que nous adopterons cependant afin d'attirer l'attention sur une tension entre contenu et relation, car, dans les campus numériques, la médiatisation des contenus d'enseignement s'accompagne de la médiation entre enseignant et apprenant via la machine et le réseau. 

courrier électronique permet des échanges faciles et peu onéreux entre l'enseignant et l'étudiant. Mais ce mode d'échange est aussi (et surtout) coûteux pour l'enseignant en temps de travail. Lorsque l'activité se développe réellement, les enseignants ou tuteurs commencent à se plaindre de l'augmentation du temps de travail et posent la question du mode de rémunération. Le forum, mode d'écriture collectif, évite à l'enseignant la multiplication des échanges et des réponses personnalisées. La qualité de la relation peut s'en trouver altérée mais celle-ci évolue par le caractère collectif des échanges. Cependant, ici aussi, dès que les campus passent un certain seuil d'échanges, la question de la faisabilité se pose. Certains répondent en termes d'échanges personnalisés et se trouvent face à un problème de manque de ressources humaines ou de financements pour les rémunérer. D'autres renoncent au tutorat personnalisé au profit des échanges entre pairs via les forums.

Ces choix ou ces compromis se traduisent dans les objets techniques utilisés. Ainsi, parfois, la fonction courrier électronique est-elle absente de la plate-forme, parfois estelle délibérément supprimée parce que les acteurs le demandent. Certains évoquent la possibilité de « réifier » ou d'« objectiver » les termes des échanges, notamment ceux qui s'effectuent à travers les forums, à travers à l'archivage des échanges et le recours aux moteurs de recherche. Il y aurait alors passage de l'échange vivant à un contenu objectivé. Mais ce type de glissement peut-il porter sur autre chose que sur des savoirs académiques ou des procédures méthodologiques? Comment le relationnel en définitive est-il traitable et traité ? Certains campus, on l'a dit, ont été amenés à distinguer plusieurs types de relationnel (direction/tutorat/animation), ce qui montre que la question devient cruciale: chacun de ces types peut en effet faire l'objet d'une transformation en document (rubrique d'aide, mode d'emploi, guide méthodologique, etc.) ou être confié à un opérateur humain.

\section{Construire les connaissances par l'échange}

Dans une autre perspective, enfin, si la représentation dominante est celle du réseau de communication et d'échanges, les savoirs, et surtout la construction des connaissances peuvent passer, non pas seulement par des documents et la communication autour de ces derniers, mais surtout par les échanges : échanges directs entre enseignants et étudiants mais aussi échanges entre étudiants eux-mêmes. Les choix peuvent être alors ceux du travail collaboratif (où les étudiants sont amenés à construire collectivement leurs connaissances, avec ou sans l'enseignant). La tentation extrême serait alors de n'avoir plus aucun contenu objectivé en document comme ce fut le cas, du moins dans sa phase de démarrage, pour le campus Tutelec ${ }^{12}$ et de faire reposer l'essentiel de la formation sur la recherche et/ou le traitement d'informations par les étudiants à travers la réalisation de mémoires collectifs ou de projets professionnels.

Il convient donc de s'attacher aux glissements qui peuvent se produire entre information (contenu) et communication (relation) dans les dispositifs de formation à distance et à la dimension économique info-communicationnelle que ceux-ci peuvent revêtir. L'hypothèse est que sont à l'œuvre dans les campus des dynamiques qui oscillent entre la tentation de fixer des éléments communicationnels en documents (informationnels) et celle de faire passer des éléments informationnels de la forme de documents à celle d'échanges entre les participants (étudiants et enseignants). Ces dynamiques ne sont pas

Études de communication, Numéro spécial | 2007 
portées par une volonté d'ingénierie spécifique mais prennent forme à travers un tâtonnement collectif et résultent autant des contraintes techniques ou financières que des représentations et des tensions entre les acteurs.

\section{Contraintes techniques, dynamiques organisationnelles et perspectives communicationnelles}

37 Pour terminer et élargir le panorama esquissé dans cet article, il faut rappeler que l'Internet connaît aujourd'hui deux modes de développement parallèles : d'un côté le peer to peer, mode d'échanges communautaire de pair à pair par interconnexion des machines, et, de l'autre, les serveurs et plates-formes qui jouent un rôle de point de passage obligé dans la communication. Sur un plan purement théorique, les deux modes de développement peuvent concerner l'enseignement et la formation. Tout n'est cependant pas possible dans les institutions, les universités, ou du moins certains acteurs en leur sein, mettant notamment en avant les questions de sécurité des données et de protection des réseaux et ne souhaitant pas recourir au peer to peer. Les plates-formes semblent s'imposer "naturellement » comme la solution technique à la formation via Internet. Elles sont en fait le résultat d'un compromis entre acteurs : ceux que le développement des plates-formes intéresse fortement (souvent des passionnés d'informatique) et les responsables de la sécurité des réseaux informatiques. Même si des tensions se font jour, le compromis est possible, alors qu'il ne l'est pas à propos du peer to peer. La communication par l'intermédiaire des plates-formes et celle qui passe dans le peer to peer , s'inscrivent par ailleurs dans des perspectives économiques totalement différentes. Techniquement, le " podcasting " peut permettre à l'étudiant d'enregistrer le cours d'un enseignant et de le déposer sur son Blog à destination de l'ensemble d'une promotion ou même de l'Internet tout entier. Le numérique permet ainsi un nouveau type de prise de notes ${ }^{13}$. D'autres dynamiques pourraient ainsi succéder à celles des campus qui relèvent encore pour le moment des représentations dominantes de l'enseignement dans le monde universitaire.

Sur le plan communicationnel, il semble nécessaire de garder une certaine distance par rapport à la perspective d'un développement important des interactions personnelles entre des enseignants autonomes très qualifiés et des étudiants. En effet, l'université s'est massifiée depuis 20 ans et on y distingue une tension très nette entre d'une part la gestion de cohortes très nombreuses, notamment dans les premières années du niveau L où les interactions sont relativement faibles, et les niveaux $\mathrm{M}$ et $\mathrm{D}$ où les effectifs sont faibles et l'encadrement très personnalisé. Sur un plan communicationnel, il semble que le modèle qui émerge des différents dispositifs et campus soit en fait assez proche de celui de l'enseignement mutuel du XIX ${ }^{e}$ siècle: les contenus d'enseignement transférés sur documents sont confiés à des moniteurs qui ne sont pas (ou pas encore) des universitaires au sens strict.

Sur un plan informationnel, il semble que les thèses sur la non-rivalité de l'information ne tiennent pas compte de la réalité socio-économique des établissements d'enseignement supérieur ${ }^{14}$. En théorie, en effet, toutes les ressources intellectuelles produites par une université pourraient être mises gratuitement à la portée de tous. En terme d'éthique, cela correspondrait même à une volonté d'accroissement de la connaissance générale indépendamment de tout intérêt matériel. D'un point de vue économique purement théorique, cela ne devrait pas non plus léser les établissements 
dans la mesure où ceux-ci ne sont pas des éditeurs, leur activité principale étant de délivrer des diplômes à partir de l'enseignement dispensé et de l'évaluation du degré d'assimilation de ces savoirs par les étudiants. Mais raisonner ainsi, c'est ne pas tenir compte d'un facteur important qui est la rivalité entre les universités. Certes, en France aujourd'hui, celle-ci ne se joue pas en termes marchands, car on reste dans un contexte où l'essentiel du financement des universités publiques vient de l'État, sur la base de critères normés. Mais au moment où le nombre d'étudiants stagne et commence même à baisser, attirer un plus grand nombre d'étudiants redevient un enjeu et ne pas mettre en accès libre des contenus d'enseignement est perçu comme un élément stratégique par une majorité d'enseignants totalement étrangers aux nouvelles dynamiques de l'Internet.

\section{Conclusion}

L'université est restée longtemps hors des tendances industrialistes en éducation. Face à la massification elle ne sait que multiplier les groupes d'étudiants et reste une activité de main d'œuvre. L'étude des campus soulève la question des tensions qui s'installent et des tendances qui se dessinent à travers les dispositifs numériques de formation à distance. L'enseignement à distance de niveau Licence tendrait ainsi à s'industrialiser de manière classique par diffusion de produits de formation standardisés accompagnés d'un encadrement qualifié minimal, comme jadis dans le modèle de l'enseignement mutuel. Par contre, au niveau du master, niveau dans lequel les universitaires se reconnaissent davantage comme enseignants-chercheurs, le modèle universitaire classique reste dominant: des chercheurs, producteurs du savoir, entrent dans une relation de type personnel avec des étudiants considérés comme disciples ou futur collègues. Pour reprendre la terminologie de Boltanski et Thévenot évoquée en introduction, on ne se situe pas ici dans un cadre marchand ou industriel, ni non plus tout à fait dans le cadre civique : on serait plus dans une perspective de type domestique, voire artiste.

Sur la période 2000-2005, le numérique n'a donc pas bouleversé l'institution universitaire qui est confrontée à un problème d'effectifs et de répartition de moyens d'un tout autre ordre. Les dispositifs numériques de formation semblent n'avoir en perspective à court terme qu'un enseignement de type mutuel pour les cycles massifiés et le maintien du mode individuel pour les niveaux supérieurs conduisant à la recherche. Ce faisant, même s'ils ne sont pas parvenus à inventer de nouvelles formes de communication pédagogique, ils actualisent à nouveau la question centrale de l'industrialisation de la formation : celle de la part respective de l'enseignant et du document dans les dispositifs de formation et de leur possible ré-articulation à travers les objets techniques.

\section{BIBLIOGRAPHIE}

Deceuninck, J., (2005), Campus Forse, Formation et Ressources en Sciences de l'éducation, Accessible aux adresses indiquées à ERTe CN 2005. 
Deceuninck, J. et Laisne, M., (2004), Vers une ingénierie des dispositifs innovants dans la formation des enseignants, in : Cahiers d'étude du CUEEP, Innover en formation des maîtres et des formateurs d'adultes, USTL-CUEEP, $\mathrm{n}^{\circ}$ 54, juin 2004, pp. 11-17.

ERTe CN, (2005), Résultats 2005 de l'ERTe (Équipe de Recherche Technologique éducation) « Modèles économiques et enjeux organisationnels des campus numériques ", accessible à www.ifresi.univlille1.fr/SITE/2_Recherche/22_Programmes/ERTe/ERTe.htm ou à partir de la page d'accueil www.mshparisnord.org/.

Fichez, E. et Benchenna, A., (2005), Modèles économiques et enjeux organisationnels des Campus numériques : le cas CampusCultura, accessible aux adresses indiquées à ERTe CN 2005.

Garcia, C., (2003), « Croyance pédagogique et innovation technologique. Le marché de la formation à distance au service de la 'démocratisation' de l'enseignement supérieur ", in : Les actes de la recherche en sciences sociales, septembre 2003.

Grevet, P., (2005), Modèles économiques et enjeux organisationnels des Campus numériques : L'expérience économico-institutionnelle de Canège, accessible aux adresses indiquées à ERTe CN 2005.

Grevet, P., (2006), Le numérique dans l'enseignement supérieur : des tendances néo-servicielles, in : Éducation Permanente, $\mathrm{n}^{\circ}$ 169, 2006-04.

\section{NOTES}

1. Présentation de l'étude de l'ERTE (2005).

2. Ceux-ci n'ont pas relevé des études de l'ERTe.

3. Il s'agit du Campus Pégasus. Ce projet, qui se présente comme «Portail de reprise d'études graduelle et d'accès par un soutien personnalisé à l'université ", regroupe 6 universités et le CNED.

4. A titre d'exemple, on peut citer Paris 3 et son site «Télé 3 Sorbonne nouvelle » qui propose également une offre de formation.

5. La concurrence reste, certes, limitée puisque les publics en formation sont massivement régionaux. Mais l'individualisme des organismes de formation est patent.

6. On pourrait employer ici le terme de « bénéfices » si l'on en croit les déclarations des porteurs du projet. En l'absence de données chiffrées, et surtout en l'absence d'une comptabilité analytique, il est impossible de dire de fait si des bénéfices sont réellement dégagés. Ce dont on parle ici est essentiellement le résultat d'exploitation (écart entre les coûts identifiés par les acteurs et les recettes obtenues par les inscriptions des étudiants).

7. Dans la plupart des dispositifs de formation à distance, les examens continuent à se passer en présentiel dans les locaux de l'université et, dans leur écrasante majorité, sous la forme d'épreuves écrites traditionnelles. Ceci n'est pas sans effet sur les étudiants. On a pu constater dans certains cas que ceux-ci ont été amenés à demander «moins de multimédia » au profit d'une préparation sous forme de polycopiés, plus classique mais les préparant mieux, de leur point de vue, à des épreuves de type composition ou dissertation. On est ici face à une «dynamique du diplôme » sur laquelle s'accordent les différents acteurs et qui a une incidence limitative sur la médiatisation.

8. On pense notamment à l'article de C. Garcia (2003).

9. Par exemple les informations sur les dispositifs liés au CNED ne sont pas reprises dans les documents de communication et sur le site de la FIED. De même le CNED ne donne aucune information sur les dispositifs de formation à distance des universités. 
10. Mais il faut rester très prudent : les UNR et les UNT font également l'objet de traductions très diverses.

11. Le terme de « ressources " recouvre des choses très différentes, allant du polycopié en ligne sous forme de fichier PDF à des exercices de type QCM, en passant par d'authentiques réalisations hypermédia. Celui de «support " n'est guère plus précis, associant des éléments de taille et de poids différents au sein du dispositif de formation. Le terme «learning object » se veut générique en proposant une approche en termes de « granularisation » de la formation.

12. Le campus Tutelec (Tutorat électronique) visait la formation initiale de jeunes enseignants directement mis en poste après le passage du concours de professeur des écoles. Leurs besoins de formation étaient mal connus et les réponses, savoirs d'expertise d'enseignants professionnels et de formateurs, non formalisées. Dans sa première version, le campus se posait donc en point de rencontre entre demandeurs et experts. La formalisation progressive des besoins et des réponses devait faire évoluer le dispositif tout en gardant l'idée centrale de "tutorat électronique », de communication interpersonnelle individuelle et collective à distance.

13. Mais l'enseignant a plutôt tendance à le refuser car la possibilité de reproduction et de diffusion des savoirs qu'il transmet semble remettre en cause le rôle du professeur propriétaire de son enseignement.

14. P. Grevet Le numérique dans l'enseignement supérieur : des tendances néo-servicielles, in : Éducation Permanente, ${ }^{\circ}$ 169, 2006-04.

\section{RÉSUMÉS}

L'étude de dispositifs de formation diplômants qui se sont développés dans le cadre des Campus numériques montre qu'ils se sont inscrits dans une dynamique générale qui traverse le champ universitaire dans son ensemble et que celle-ci relève majoritairement des modes de pensée universitaires traditionnels. En même temps, cependant, ces campus actualisent la question relative au type d'équilibre d'ordre info-communicationnel dans les dispositifs de formation. Les formations de niveau Licence, caractérisées par des effectifs nombreux, ont tendance à recourir à des supports standardisés et sont accompagnées d'un tutorat faiblement qualifié comme dans le modèle scolaire de l'enseignement mutuel au début du XIX ${ }^{\mathrm{e}}$ siècle. Par contre, au niveau du Master, les enseignements continuent à relever du modèle universitaire traditionnel et restent étrangers à cette dynamique.

The study of diploma granting systems which have developed with the Virtual campuses framework shows that they exist within a general dynamic, that touches the entire university field, which is mainly influenced by traditional university thinking. At the same time, these campuses actualise questions related to the type of info-communicational balance necessary within these educational systems. At the licence level, the educational applications are characterised by the use of a large number of effectives and tend to use standardised applications coupled to non qualified tutorial activities, a strategy which resembles the "mutual» educational model of the early 19th century. But at the Masters level, teaching continues to rely on the traditional university mode. 
INDEX

Keywords : digital campus, training, higher education, document, information, communication, exchange

Mots-clés : campus numérique, formation, enseignement supérieur, document, information, communication, échange

\section{AUTEUR}

\section{JULIEN DECEUNINCK}

Julien Deceuninck est maître de Conférences à l'Université Lille 3, attaché au laboratoire GERIICO et membre du Séminaire Industrialisation de la Formation. Ses travaux de recherche portent sur les dispositifs de formation intégrant le numérique dans l'enseignement supérieur (campus numériques, formation des enseignants et des personnels des bibliothèques, enseignement à distance et formation continue). Adresse électronique : julien.deceuninck@univlille3.fr. 